\title{
त्री \\ El argumento del regreso del experimentador y la replicación de experimentos
}

Romina ZuPPONE

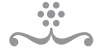

RESUMEN

El propósito de este trabajo es analizar y criticar el argumento del regreso del experimentador propuesto por Harry Collins en 1985 . Para ello, comenzaremos comentando el experimento destinado a detectar ondas de gravedad que diseñó Joseph Weber en la década de 1970. Luego, continuaremos con el análisis y la discusión de las dos versiones del argumento de Collins: la versión epistemológica y la versión ontológica. Finalmente, luego de formular los lineamientos básicos para una teoría de la reproducción experimental, y una elucidación del concepto de replicación, propondremos dos formas de evitar el regreso del experimentador.
\end{abstract}

Palabras- GLave $\bullet$ Argumento del regreso del experimentador. Experimento.

Reproducción experimental. Replicación. Collins. Weber.

Acordamos con el plan de reemplazar la unidad a partir de la predicción del fallo. Debemos advertirles, sin embargo, que nuestros hallazgos preliminares nos indican que la computadora 9000 a bordo, ha predicho erroneamente el fallo. Insisto: la predicción es errónea. Sé que esto puede parecer increíble, pero esta conclusión se basa en los resultados de la computadora 9000 melliza. Nuestro escepticismo nos lleva a realizar nuevas pruebas para determinar la validez de la conclusión (Arthur Clarke \& Stanley Kubrick, A space odyssey, 2001).

\section{INTRODUGGIÓN}

En las últimas décadas, los estudios acerca de la ciencia, tanto por parte de filósofos, como de sociólogos e historiadores, comenzaron a revelar un interés creciente por la experimentación y sus problemas. Trabajos como de Latour y Woolgar (1979) y de 
Collins (1985), y las conclusiones relativistas, a las que los autores arribaron a partir de sus estudios etnometodológicos, contribuyeron e incitaron a la reflexión respecto de la racionalidad en la práctica experimental y resultaron un estímulo especialmente para la filosofía de la ciencia, que había tradicionalmente asumido la relevancia de la experimentación y de sus resultados en la constitución del conocimiento científico.

Desde entonces, un conjunto de preguntas ha convocado a los epistemólogos y ha permitido delinear la agenda de problemas en el ámbito de la filosofía de la experimentación. En primer lugar, se procuró un relevamiento de carácter empírico de las funciones que los experimentos desempeñan en las ciencias naturales (cf. Hacking, 1996 [1983]), cuyo objetivo principal era trascender las limitaciones de las propuestas epistemológicas de la concepción heredada, que consideraban al experimento sólo como un medio para contrastar teorías científicas (cf. Popper, 1959), y a su vez, las limitaciones de las propuestas de la epistemología historicista que, si bien atribuía a la experimentación numerosas funciones, negaba que la contrastación de teorías científicas fuese una de ellas (cf. Kuhn, 1977). En segundo lugar, se consideró necesario dar cuenta de la dinámica que se establece entre instrumentos, datos y teorías, permitiendo comprender en qué radica la estabilidad de la base empírica y qué clase de dependencia teórica es posible atribuir a los datos experimentales, permitiendo este punto del programa experimentalista contribuir a la reflexión respecto de los procesos de continuidad y de ruptura en las ciencias naturales (cf. Ackermann, 1985; Hacking, 1992). En tercer lugar, los filósofos del experimento procuraron tomar posición respecto de las razones por las cuales un resultado experimental es aceptado; frente a la pregunta respecto de si acaso la aceptación de un resultado experimental depende de factores de carácter social y económico o, por el contrario, de la satisfacción de criterios epistemológicos internos a la práctica científica, se inclinaron por la segunda alternativa defendiendo la racionalidad de la práctica experimental y proporcionando para ello diferentes razones, entre ellas, por ejemplo, la aplicación de lo que Allan Franklin denominó estrategias epistemológicas, es decir, el empleo de un conjunto de criterios internos a la práctica que permiten argumentar respecto de la corrección de los resultados obtenidos en el transcurso de un experimento (cf. Franklin, 1989, 1998; Radder, 1992, 2003; Hones, 1990; Culp, 1995). Finalmente, en el contexto de los problemas relevantes a la filosofía del experimento y en relatar con lo anterior, se procuró dar cuenta de cómo se satisface la norma de intersubjetividad de la práctica experimental, es decir, si los resultados experimentales son aceptados en virtud de que son reproducibles, entonces ¿qué significa reproducir un resultado experimental? (cf. Radder, 2003).

Los últimos dos problemas de la agenda de la filosofía de la experimentación recibieron atención creciente a partir de los resultados de la sociología del conocimien- 
to científico, en especial, de ciertas conclusiones a las que arriba Collins (1985). Como tendremos oportunidad de evaluar en las próximas páginas, Collins propone, a partir de sus estudios de campo en el ámbito de los experimentos que tenían por objeto evaluar la existencia o no de radiación gravitatoria, que lo único que puede establecer un resultado experimental son elementos externos al experimento, elementos relacionados fundamentalmente con la capacidad de persuasión de los actores involucrados.

Por lo tanto, frente a las preguntas - ¿cómo decidir si un resultado experimental obtenido en la investigación de frontera es correcto? ¿Qué papel desempeña la replicación en estas situaciones? ¿Qué inconvenientes se presentan cuando intentamos determinar un resultado desconocido a partir de un instrumento diseñado específicamente para tal propósito? - Collins adopta una actitud escéptica y relativista. Esta posición se sostiene a partir de su formulación del argumento del regreso del experimentador: un argumento que busca poner de manifiesto que, en la actividad experimental, la determinación de un resultado desconocido, por medio de un instrumento diseñado para tal fin, no puede justificarse racionalmente. Entiende Collins que la presencia de formas de argumentación inválidas en el proceso de establecimiento de la evidencia empírica (en sentido lógico) obligaría a determinar cuál es el resultado correcto de un experimento apelando a factores de carácter externo a la práctica científica, factores no-epistémicos, como, por ejemplo, sociales o económicos (cf. Collins, 1981, 1985, 1994, 2002).

Entendiendo, por el contrario, que la práctica experimental provee conocimiento confiable, que la justificación de los resultados está racionalmente garantizada, pero entendiendo, al mismo tiempo, que el argumento del regreso del experimentador cuestiona estas intuiciones, buscaremos en este trabajo indicar en qué consiste dicho argumento, determinar cuál es el desafío que plantea a la racionalidad en la práctica experimental y proponer algunas respuestas y objeciones al mismo. Para ello, la estrategia es la siguiente: en primer lugar, ofreceremos una introducción a los primeros intentos dirigidos a detectar ondas de gravedad, un caso del que Collins se vale para ilustrar su tesis. Luego, analizaremos y discutiremos los argumentos de Collins y los presupuestos involucrados en ellos. Por último, propondremos una respuesta de carácter filosófico que permite, según creemos, eludir el argumento del regreso del experimentador y, a la vez, proporcionar una comprensión más rica y adecuada de la práctica experimental y de sus problemas. 


\section{LA DETEGGión DE LA RADIAGIÓN GRAVITATORIA EN 1970}

Collins formula el argumento del regreso del experimentador tomando como caso paradigmático la investigación acerca de la existencia de ondas de gravedad. Según entiende, la controversia sobre la existencia de altos flujos de ondas gravitatorias no pudo dirimirse a partir de criterios epistémicos, sino que el fin del conflicto - el quiebre del regreso - se logró sólo a partir de las dotes retóricas de uno de los investigadores involucrados en el caso, Garwin. Considerar este episodio, al menos brevemente, nos permitirá una comprensión más ajustada de la controversia y del alcance de las conclusiones de Collins.

Se deduce de la teoría de la relatividad general que los cuerpos masivos, al ser acelerados, deforman el espacio-tiempo curvo. A su vez, esta deformación geométrica, que se propaga con la velocidad de la luz, se manifiesta como una oscilación del espacio-tiempo, como una onda de gravedad que transporta energía, la cual puede ser medida por detectores muy sensibles.

Los fenómenos que producirían radiación gravitatoria son numerosos: las explosiones de novas y supernovas, la formación de agujeros negros y las colisiones entre éstos, los púlsares binarios, las estrellas de neutrones y la formación del espaciotiempo a partir del Big Bang son algunas de las fuentes conocidas de ondas gravitatorias. Conocer la fuente de la radiación permite acotar el espectro de frecuencias previsibles para las ondas y construir consecuentemente el detector más apropiado para el tipo de fuente cuya radiación se intentará captar (cf. Davies, 1987 [1980], p. 77 ss.).

Joseph Weber, físico e ingeniero, se dedicó, durante la década de 1960, a construir un detector de ondas gravitatorias (cf. Weber, 1960, 1967, 1968a, 1968b, 1972). El detector de Weber no es un aparato excesivamente sofisticado: consiste en un cilindro macizo de aluminio, de $1.53 \mathrm{~m}, 0.66 \mathrm{~m}$ de diámetro y 1,4 , toneladas, que funciona al modo de un oscilador armónico sintonizado para captar la radiación gravitatoria de frecuencia cercana a los $1660 \mathrm{hz}$, la cual correspondería a la radiación emitida en los colapsos de supernovas (cf. Weber, 1969). La antena, a su vez, lleva conectados transductores que convierten las oscilaciones de la barra en impulsos eléctricos, los cuales son posteriormente amplificados y registrados (cf. Davies, 1987 [1980]).

Las ondas de gravedad - que pueden concebirse como análogas a las ondas electromagnéticas - transportan muy poca energía, de modo que la señal que producen en un detector es sumamente débil; por consiguiente, el problema fundamental para quienes intentaron detectarlas radicó en cómo distinguir la señal causada por la vibración, que una onda produciría en el detector, de las vibraciones causadas por otras fuentes. Para minimizar el problema del ruido, Weber tomó numerosas precauciones: los controladores utilizados (cf. fig. 1) permiten detectar de manera independiente seña- 
Figura 1. Detector tipo Weber de ondas gravitatorias. (Adaptada de Collins, 1985, p. 82).

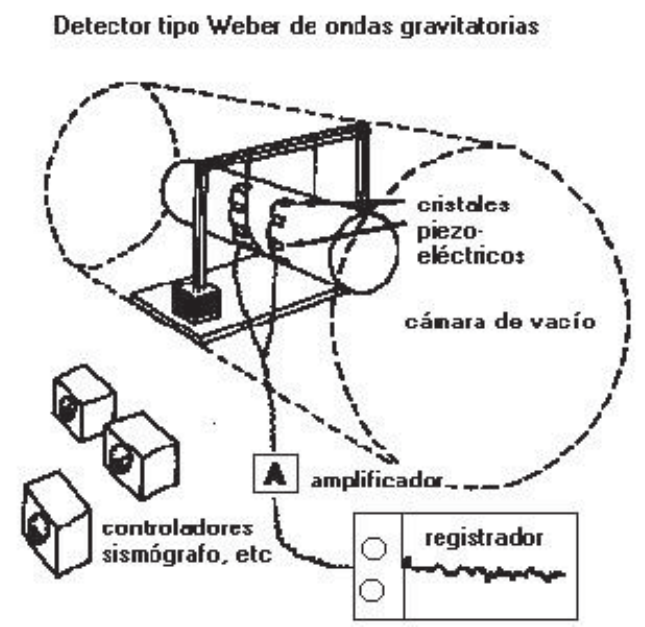

les que la antena captaría como ondas gravitatorias, no siendo estas más que producto de movimientos sísmicos o de ondas electromagnéticas. Al ser el ruido térmico ineliminable, ${ }^{\mathbf{1}}$ Weber fijó el umbral a partir del cual las oscilaciones detectadas podrían atribuirse a ondas gravitatorias. Sólo tras haber tomado tales precauciones, anunció los resultados obtenidos: sus registros indicaban al menos 7 pulsos diarios que no podían atribuirse más que a ondas gravitatorias. Weber (1969) dio a conocer estos resultados. Es importante destacar que dicho exceso -interpretado como la detección de altos flujos de ondas gravitatorias de frecuencia cercana a los $166 \circ \mathrm{Hz}-$ si bien no es incompatible con las teorías físicas y cosmológicas aceptadas, es altamente improbable a la luz de éstas, razón por la cual fue recibido con gran escepticismo. Tal como afirma Levine, colaborador de Garwin en la detección de radiación gravitatoria:

La emisión de energía gravitatoria equivalente a 5 masas solares, diariamente, de una fuente alejada $3 \circ 0$ años luz de la Tierra, sería insuficiente para producir los resultados que obtuvo Weber. Y un evento tal sería visible a plena luz del día (Levine, 2004, p. 45).

Posteriormente, Weber anunció la simultaneidad entre señales detectadas por dos detectores alejados (cf. Weber, 1968a), y consideró que ambas eran producto de una única fuente en el centro de la galaxia (cf. Weber, 1970). Y, dado que los picos detectados

1 El ruido térmico surge por los movimientos moleculares, los cuales sólo cesan a $0^{\circ}$ Kelvin. Mantener el detector a tal temperatura resultaba en ese momento técnicamente imposible. Sin embargo, entre 1970 y 1990 , se han puesto en funcionamiento barras criogénicas de gran sensibilidad, capaces de reducir considerablemente el ruido termal (cf. Collins, 2004). 
tenían un período de veinticuatro horas, anunció haber detectado una correlación sideral. Por ello, varios equipos de investigación buscaron contrastar las afirmaciones de Weber (cf. Garwin \& Levine, 1974), diseñando instrumentos similares a la antena original aunque con mayor sensibilidad, pero los resultados fueron negativos. ¿Qué decisión debe tomarse en este caso? ¿Detectó Weber ondas gravitatorias? O, por el contrario, ¿disconfirmaron los restantes equipos sus resultados? ¿Cómo determinar qué experimento fue competentemente realizado? ¿Obedece la discrepancia a sutiles diferencias entre los equipos utilizados? ¿Qué evidencia permitiría dirimir esta cuestión?

\section{El ARGUMENTO DEL REGRESO DEL EXPERIMENTADOR}

Collins (1975) ofrece un análisis de los problemas epistemológicos asociados a la investigación de la existencia o no de radiación gravitatoria. En ese artículo, sienta las bases para lo que denominará, en 1985 , el regreso del experimentador. ${ }^{2}$ En esta obra, afirma que el argumento del regreso del experimentador:

[ARE 1] Es una paradoja que se presenta a aquellos que pretenden utilizar la replicación como un test de la verdad de una proposición científica. El problema es que, dado que la experimentación es una cuestión de habilidad práctica, nunca es claro si un segundo experimento fue lo suficientemente bien realizado como para contar como una contrastación de los resultados del primer experimento. Un experimento posterior es requerido para evaluar la calidad del último, y así... (Collins, 1992, p. 2).

Según Collins, si hemos de determinar el valor de verdad de un enunciado empírico obtenido por vía experimental, debe apelarse a la replicación. Lamentablemente, a pesar de los numerosos artículos dedicados al tema, Collins no proporciona una definición satisfactoria del término. Él parece asumir que un experimento es la replicación de otro si los mismos son idénticos, sin indagar a qué clase de identidad se está refiriendo. Podríamos asumir a partir de ciertas citas que el autor se refiere a la identidad funcional, por ejemplo, al decir:

2 En este artículo, utilizamos la segunda edición de la obra, publicada en 1992, que introduce un epílogo en el que Collins recupera algunas de las críticas que recibió su trabajo. En lo sucesivo, abreviamos o argumento del regreso del experimentador, por ARE. 
Discutiendo los intentos de detección de altos flujos de ondas gravitatorias a comienzos de 1970, un físico ilustró la situación comentando que tal vez la diferencia entre un aparato y el otro se debiera a que el primero apoyó sobre los transductores una copia del Physical Review Letters mientras el pegamento se secaba (Collins, 1991, p. 135).

Si aparentemente se cumple la identidad material entre detectores, detalles presuntamente triviales, como el explicitado en la cita anterior, pueden dar como consecuencia que el primer y segundo detector no sean funcionalmente idénticos, y esta diferencia se debería a la existencia de conocimiento tácito, esto es, conocimiento no-proposicional que, aun desempeñando un papel importante para el funcionamiento del instrumento, no puede transmitirse por los medios convencionales. Si bien la noción de conocimiento tácito fue acuñada por Polanyi (1967), Collins define el conocimiento tácito como:

Conocimiento o habilidades que pueden ser traspasadas entre científicos por contacto personal pero que no pueden ser, o no han sido, transferidos por medio de fórmulas, diagramas o descripciones verbales así como tampoco por medio de instrucciones para actuar (Collins, 2001, p. 72).

Entonces, si los dispositivos no son funcionalmente idénticos, las diferencias en los resultados obtenidos en ambos experimentos pueden obedecer a diferencias en los instrumentos de detección, con lo que, según Collins, no sería posible dirimir qué enunciado empírico es verdadero.

En una segunda aproximación al problema de la validación de resultados experimentales, Collins formula el argumento del regreso del experimentador de esta manera:

[ARE 2] Cuál sea el resultado correcto depende de que efectivamente haya ondas de gravedad golpeando la Tierra en flujos detectables. Para resolver esto, debemos construir un buen detector de ondas de gravedad y echar un vistazo. ¡Pero no sabremos si hemos construido un buen detector hasta haber probado y obtenido el resultado correcto! Pero no sabemos cuál es el resultado correcto hasta... y así ad infinitum (Collins, 1992, p. 85).

Ciertamente, concederíamos que el resultado correcto de un experimento, tendiente a determinar la existencia de un fenómeno, evento, entidad o proceso, depende de la existencia del fenómeno, evento, entidad o proceso en cuestión. También aceptaríamos que un buen detector será aquel que provea un resultado positivo en caso de 
que el fenómeno exista, y un resultado negativo en caso contrario. Resta evaluar si concederemos que la determinación del resultado correcto se basa únicamente en el buen funcionamiento del detector. ${ }^{3}$

Las dos versiones del ARE que hemos reproducido pretenden poner en cuestión la racionalidad de la práctica experimental, ya que, según Collins, los recursos experimentales nunca son suficientes para establecer un resultado; por el contrario, aquello que es determinante y que permite superar el regreso del experimentador está directamente relacionado con factores no-epistémicos. En el caso que aquí presentamos, Collins afirma que Garwin y su colaborador, Levine, lograron persuadir a la comunidad científica respecto de la inadecuación del trabajo de Weber; lo que significa que la persuasión no fue alcanzada sólo por medio de la aplicación de recursos epistémicos. En sus palabras:

He sugerido que todas estas tácticas de negociación son intentos por quebrar el regreso del experimentador. Deben emplearse tácticas no-científicas porque los recursos del experimento no son suficientes (Collins, 1992, p. 14,3).

Entonces, ya sea en virtud de que el único modo de reproducir un resultado (y, con ello, la satisfacción de la norma de intersubjetividad de la práctica experimental) es replicando un experimento, pero mostrando que esto último no es posible, como sucede en ARE 1, o ya sea en virtud de mostrar que no es posible un acceso al mundo empírico teóricamente independiente y que la posibilidad de justificación de un resultado experimental depende de eso, como se intenta con ARE 2, Collins deja planteado un problema para el que pueden proponerse dos clases de soluciones: la que él adopta, es decir, aquella solución que quiebra el regreso apelando a razones de carácter externo a la práctica científica, o aquella que intentaremos defender en este trabajo, que insistirá en los diversos modos de satisfacer el criterio de intersubjetividad de la práctica científica, defendiendo la importancia del conocimiento teórico en la interpretación de los resultados experimentales, como veremos especialmente en la quinta sección de este trabajo.

3 La constitución de un resultado experimental es un proceso complejo, del que no podremos dar cuenta en detalle en este artículo, pero sí mencionaremos algunos aspectos relevantes para ese trabajo. Podríamos afirmar que los experimentos producen outputs, eventos que son directamente perceptibles por el observador, por ejemplo, el movimiento de una aguja en un voltímetro, el sonido en un contador de partículas Geiger etc. Ahora bien, en los casos en los que el resultado final del experimento sea subsumible bajo un concepto métrico, el output adquirirá parte de su significado al establecer una relación con una magnitud, por un proceso que podemos denominar interpretación interna (en el sentido en que es interna al experimento). Como consecuencia de esta interpretación, obtendremos datos que serán sometidos al análisis estadístico pertinente para el tipo de experimento, obteniéndose, al finalizar ese proceso, un resultado que será expresado con su margen de error. 
Presentadas ya ambas versiones del ARE, intentaremos a continuación analizarlas, determinar cuáles son los presupuestos que permiten la formulación de ambas versiones y establecer en qué se diferencian. Aunque Collins parece asumir que ARE 2 es tan sólo un caso particular de ARE 1, entendemos, sin embargo, que, si bien en ambos casos se pone de manifiesto un problema de carácter epistémico, por cuanto los argumentos revelan las limitaciones de nuestros medios para obtener conocimiento científico y abordar el mundo físico, en el caso ARE 1, que en lo sucesivo denominaremos versión epistemológica, el énfasis recae en el problema de la contrastación de resultados experimentales, en la relevancia de la replicación de los experimentos, un problema que a su vez está vinculado con la postulación de juicios de identidad; mientras que en la segunda formulación (ARE 2), al afirmar que el único modo de determinar el resultado correcto es a partir de un instrumento que funcione adecuadamente, se relaciona, por el contrario, con la tesis de la autonomía de la experimentación. Ya que, al pretender reconocer el resultado correcto a partir de la idoneidad de un dispositivo de detección de una señal variable y débil, se desestima la importancia del contexto teórico en el que el experimento se desarrolla y a partir del cual adquiere significado, se minimiza el valor del conocimiento aceptado y el hecho de que este conocimiento es el que da forma y sentido a la investigación, delimitando las respuestas posibles. Es por ello que la aceptación de ARE 2 nos compromete con una versión extrema de la tesis de la autonomía de la experimentación, al suponer que el reconocimiento de la corrección de un resultado experimental depende exclusivamente de cuestiones empíricas, en particular, las relativas al funcionamiento de los instrumentos. Teniendo en cuenta que la segunda formulación del argumento supone que la determinación del resultado correcto es sólo producto de la captación de una relación entre objetos del mundo, creemos pertinente denominarla versión ontológica.

Godin y Gingras (2002) aducen que el ARE no es sino una reformulación del argumento escéptico respecto de la fundamentación del conocimiento propuesta por Montaigne, quien retomando el desafío de Sexto Empírico, afirma:

Para juzgar las apariencias que recibimos de los objetos, necesitamos instrumentos de juicio; para verificar el instrumento, necesitamos una demostración; para verificar esta demostración, un instrumento: nos encontramos en un círculo (Montaigne, 1862, p. 544).

Es preciso en este punto detenernos en ambas versiones del ARE para hacer algunas precisiones más. Tal como es formulada, la versión epistemológica tiene como objetivo mostrar que, en el establecimiento de la evidencia experimental, se incurre en una regresión al infinito. Según esta versión, para aceptar un resultado experimen- 
tal como evidencia empírica, éste debe ser reproducido. Esta reproducción se llevará a cabo por medio de la replicación del experimento original, sin embargo, tampoco existe la certeza de que el segundo experimento sea realizado apropiadamente, de modo que se vuelve necesaria una reproducción posterior, y así sucesivamente. Algo análogo sucede en el caso de la calibración, cuando ella es relativa a otros instrumentos. En estos casos, el instrumento se calibra respecto de otro que, a su vez, depende de la calibración de otro instrumento, y así sucesivamente. Ahora bien, la versión ontológica del ARE no adopta la misma forma argumentativa: ahora, determinar si existen o no ondas de gravedad depende de la decisión respecto de si el instrumento utilizado es o no es un buen detector de ondas de gravedad, pero esta última decisión depende a su vez de la determinación previa de la existencia de las ondas. En este caso, el argumento adopta la forma de un argumento circular de tipo recíproco, en el cual, con el propósito de probar una cierta conclusión, se asume como verdadera una premisa cuya verdad, a su vez, depende de la verdad de la conclusión que se intenta probar (cf. Barnes, 1990).

En general, los autores que han criticado las afirmaciones de Collins, ${ }^{4}$ particularmente aquellos que se han detenido en el análisis del argumento del regreso del experimentador, han sostenido que ARE 1 y ARE 2 son formulaciones diferentes del argumento, sin embargo, ningún autor explica en qué radica la diferencia, ni cuál es el nexo entre ambas. Tal es también el caso de Radder, quien tras haber citado ambas versiones, afirma:

Estas dos formas de poner en cuestión el poder (dis)confirmatorio de la replicación llevan a dos formulaciones diferentes del regreso del experimentador, ambas presentes en el libro de Collins, aunque no sean distinguidas explícitamente (Radder, 1992, p. 67).

Pero, ¿qué debemos entender aquí por diferente?Y, ¿cuál es el vínculo, si lo hubiera, entre las dos formulaciones? Volveremos sobre este aspecto en las próximas secciones.

Collins (1992, cap. 2) analiza los diversos modos de satisfacer la condición de intersubjetividad en la práctica científica y los problemas que se presentan al intentar cumplir con tal requisito. He aquí una reconstrucción de las ideas contenidas en dicho capítulo:

4. El argumento de Collins ha recibido numerosas críticas desde diversos ángulos, nosotros analizaremos el argumento con el fin de mostrar un non-sequitur. Véase además de los autores ya mencionados, Culp (1995). Para una discusión respecto de la inconsistencia entre el empirismo y el relativismo en el pensamiento de Collins, véase Laudan (1982). Para una crítica del argumento desde la sociología de la ciencia, véase Knorr-Cetina (1982). 
(1) Un resultado experimental, para ser científicamente significativo, debe poder repetirse, existiendo diversos medios para la consecución de tal fin:

(a) la repetición en la misma realización material, esto es, por sucesivas secuencias experimentales llevadas a cabo con el instrumento original;

(b) por medio de la replicación del equipo original, esto es, realizando copias del instrumento;

(c) por confirmación independiente, es decir, valiéndose de instrumentos cuyo funcionamiento esté basado en principios físicos diferentes. 5

(2) Los modos anteriormente mencionados para repetir un resultado experimental no son equivalentes respecto del grado de confirmación o disconfirmación que proveen. Según Collins, la replicación aporta mayor grado de disconfirmación y un experimento basado en principios físicos independientes aporta mayor grado de confirmación en caso de proveer igual resultado. Ello es así por las siguientes razones:

(a) Las secuencias sucesivas en un mismo aparato no aportan evidencia respecto de la corrección del resultado. Dice Collins, retomando a Franklin y Howson (1984):

Para que un experimento constituya un test de otro anterior no debe ser exactamente el mismo, pero tampoco muy diferente. Tómese un par de experimentos, uno que origine un nuevo resultado y un experimento subsiguiente. Si el segundo experimento es demasiado similar al primero, no agregará ninguna información confirmatoria. El caso extremo, en el que cada aspecto es literalmente idéntico al primero, no es siquiera un experimento separado. En estas circunstancias, el segundo experimento no significará más que la lectura del informe experimental una segunda vez (Collins, 1992, p. 34).

Sin embargo, el autor acepta que "en un área poco comprendida, los científicos (...) no son capaces de garantizar que los resultados serán los mismos y entonces la observación de estos resultados sí agrega confirmación” (Collins, 1992, p. 35).

5 En su investigación, Collins omite el caso de instrumentos del mismo tipo - de hecho, el caso más usual en las ciencias empíricas - que no pueden entenderse como copias, puesto que contemplan ciertas variaciones que permiten incrementar su sensibilidad respecto del original. Volveremos sobre este punto en el apartado 3 esbozando criterios que nos permitirán esclarecer las expresiones "mismo tipo que", "copia", "réplica” y expresiones relacionadas con estas. 
(b) La replicación es problemática cuando se desconocen las posibles fuentes de error que pueden enmascarar o simular el fenómeno que se investiga. Dice al respecto:

En ausencia de un conjunto bien establecido de variables relevantes, cualquier cambio en la situación experimental, sin importar cuan trivial en apariencia, bien puede implicar cambios significativos, aunque invisibles, en las condiciones (Collins, 1992, p. 35).

(c) La contrastación independiente, si bien permite confirmar un resultado, no es aplicable como medio para disconfirmarlo, dado que podrían omitirse elementos relevantes que incidieron en la detección por la vía original. "Si el segundo experimento no provee el resultado pretendido, diferencias en el diseño entre el primero y el segundo pueden ser invocadas como la causa de la falla" (Collins, 1992, p.36).

(3) Teniendo en cuenta que no toda forma de contrastación de una hipótesis de bajo nivel suministra un grado equivalente de confirmación o disconfirmación de la proposición en cuestión, no todos los medios anteriormente mencionados son vías legítimas para la confirmación o disconfirmación del resultado original.

(4) Llegado a este punto, Collins concluye que el único medio legítimo para disconfirmar un resultado, al menos en investigación de frontera, es la replicación. "La fuerza de la disconfirmación asciende en tanto el segundo experimento se aproxima a la identidad con el primero" (Collins, 1992, p. 36).

(5) Dado que la replicación involucra conocimiento tácito, no es posible ofrecer en un conjunto finito de pasos instrucciones para obtener una réplica del diseño original.

La habilidad experimental tiene el carácter de un talento que sólo puede ser adquirido y desarrollado con la práctica. En tanto es un talento, no puede ser completamente explicado o absolutamente establecido. [...] La habilidad experimental es invisible en su pasaje y en aquellos que la poseen (Collins, 1992, p. 74). 
(6) Además, la determinación del resultado correcto del experimento, así como del buen funcionamiento del instrumento y del experimentador experto son propiedades que se determinan mutuamente. "El funcionamiento del aparato, partes del aparato y el experimentador son definidos por la habilidad de formar parte en la producción del resultado experimental correcto. Otros indicadores no pueden encontrarse" (Collins, 1992, p. 74).

(7) Finalmente, la ausencia de criterios de experticia, ${ }^{\mathbf{6}}$ que permitan establecer qué aparato funciona apropiadamente y cuál es el resultado correcto, hace recaer la aceptación de un resultado experimental en factores no epistémicos, como, por ejemplo, la capacidad persuasiva de los actores involucrados, los intereses creados respecto de los resultados etc.

En la reconstrucción del argumento, encontramos dos conceptos en los que habremos de detenernos: en primer lugar, el concepto de replicación y, relacionado con éste, la habilidad práctica que, según Collins, involucra conocimiento tácito. Si el único modo de reproducir un resultado experimental reside en replicar el experimento que da origen a dicho resultado, si, a su vez, el conocimiento tácito es un componente de todo experimento, intransferible, puesto que no es reconocido como relevante por los mismos experimentadores y debido a la ausencia de criterios internos que permitan dirimir qué instrumento funciona correctamente, la polémica respecto del resultado se resolvería apelando a elementos externos. Con base en todo lo anterior, Collins concluye que "[la] aparente replicabilidad o no [de un resultado], que es la conclusión de tales experimentos, realmente no es una cuestión del experimento" (Collins, 1992, p. 20).

Esta presentación del ARE define la siguiente agenda de problemas a tratar: en primer lugar, debe considerarse si la replicación es el único medio para disconfirmar un resultado experimental. En segundo lugar, debe evaluarse si acaso el conocimiento tácito es intransferible entre equipos de investigación. Finalmente, es preciso estudiar si, efectivamente, no existe un modo de establecer el resultado correcto independiente de la previa posesión de un instrumento de detección adecuado. Dicho de otro modo, es preciso determinar si resultado correcto y detector apropiado son conceptos que se presuponen mutuamente. De este modo, quedan delineadas las diferentes estrategias mediante las que el regreso del experimentador podría evitarse. El regreso se evitaría si podemos probar alguna de las siguientes tesis:

6 En el caso en que los diversos equipos de investigación obtengan resultados discordantes, el problema es qué tipo de criterio puede aplicarse para decidir qué experimento, si alguno, produjo el resultado correcto. Según Collins, no podemos establecerlo a partir de elementos internos al experimento según afirma en (6), entonces, la elección entre resultados rivales se torna arbitraria según muestra ( 7$)$. 
(1) La replicación no es el medio exclusivo para la contrastación de una proposición experimental.

(2) Aun si la replicación fuese la única vía para la contrastación, el conocimiento tácito puede ser transferido entre investigadores.

(3) Aun si la replicación pudiese obtenerse, no es cierto que esta sea un medio legítimo para la disconfirmación de un resultado.

(4) Aun si la replicación fuese la única vía para la contrastación de afirmaciones controvertidas, el resultado correcto puede establecerse con criterios independientes del funcionamiento de los instrumentos de detección.

A continuación, nuestra estrategia será mostrar que puede establecerse, utilizando criterios epistémicos, que dos experimentos son réplicas, que, no obstante, la replicación no es, ni puede ser, un medio para disconfirmar un resultado experimental. Finalmente, negaremos que el buen funcionamiento de un instrumento sea el único modo de establecer el resultado correcto, para lo cual sugeriremos otras opciones que nos permitan, en la investigación de frontera, evaluar los recursos experimentales en juego y su adecuación.

\section{REPRODUGGIÓN, REPETIGIÓN, REPLIGAGIÓN: ALGUNAS PREGISIONES}

La discusión de los argumentos de Collins requiere especialmente que nos detengamos en los modos en los que un resultado experimental puede reproducirse. En esta sección, por lo tanto, analizaremos de qué modos es esto posible en la práctica experimental. Al tratar la reproducción damos por supuesto que ya se dispone de un resultado, y que el objetivo de la reproducción es la comparación de este resultado original con un nuevo resultado. Aquí presuponemos, entonces, que ya se han realizado los procedimientos internos al experimento, entre ellos, la toma de datos y el tratamiento estadístico de los mismos, para dar como producto final de este proceso un resultado expresado con su dispersión.?

\footnotetext{
7 El tratamiento de este aspecto es parte de lo que denominamos individuación de los experimentos. Detallar este programa y sus logros excede los límites del presente trabajo. Cabe mencionar, sin embargo, que este capítulo de la filosofía de la experimentación se ocupa, en primer lugar, de las diferentes clases de mediciones posibles, teniendo en cuenta las características de los fenómenos en estudio, por ejemplo, si se estudian eventos continuos (como el caso de la medición de la velocidad de la luz, o la medición de las ondas de gravedad) o eventos discretos (como la medición de la carga eléctrica del electrón). Y, además, los procesos de constitución de un resultado experimental, es decir, el proceso de interpretación y análisis que comienza por el output obtenido y finaliza con un resultado teóricamente interpretado.
} 
Estableceremos ahora algunas precisiones terminológicas. En lo sucesivo, a fin de lograr claridad en el tratamiento del tema, reservaremos el término reproducción como término general para los procedimientos que tienen como objetivo la medición de una misma magnitud o un mismo parámetro, ${ }^{\mathbf{8}}$ repetición como caso especial de reproducción de acuerdo con ciertos criterios que explicitaremos a continuación, y replicación, como un caso límite de la repetición.

Lamentablemente, a pesar de la importancia que el problema de la reproducción de resultados experimentales reviste para la filosofía de la ciencia, el concepto de repetición no ha sido investigado aún con el detalle requerido, con excepción de Franklin y Howson (1984); Hones (1990); Cartwright (1991) y Radder (1992). Sin embargo no se ha conseguido aún ofrecer un tratamiento sistemático de la reproducción experimental. De modo que resulta preciso evaluar, en primer lugar, qué entendemos por reproducción y, si es posible, ofrecer una clasificación de las variedades de reproducción, que sea a la vez epistemológicamente fértil y descriptivamente adecuada, en el sentido de no contener clases vacías, ${ }^{9}$ permitiendo entonces recuperar aquello que efectivamente se realiza en la actividad científica. Propongo que reparemos inicialmente en lo siguiente: teniendo en cuenta que satisfacer la norma de intersubjetividad en la práctica científica supone la posibilidad de la reproducción experimental, ¿qué formas adopta esa reproducción?

Si entendemos la reproducción de un resultado experimental como la medición de una misma magnitud o un mismo parámetro, entonces un resultado experimental puede reproducirse. Dado que los resultados experimentales siempre poseen un margen de error, reproducir un resultado experimental no implica obtener exactamente el mismo resultado, sino un valor en el intervalo admitido:

(1) Repitiendo un mismo procedimiento con el equipo original. Dado que permanece constante el instrumento utilizado para efectuar la medición, llamaremos a este caso I-repetición. Es preciso distinguir aquí la I-repetición de lo que tradicionalmente se denomina corrida experimental (experimental run), es decir, de las múltiples mediciones que se realizan en el transcurso de un mismo experimento, que permiten luego del tratamiento estadístico, obtener el resultado.

8 Es preciso aclarar el sentido en el que utilizaremos aquí las expresiones magnitud y parámetro. Llamaremos magnitudes a todos conceptos físicos metrizados, y parámetros, a las constantes físicas. Tanto magnitudes como parámetros adoptarán valores determinados que serán sus cantidades.

9 Radder (1992) propone una clasificación que pretende ser exhaustiva aunque gran parte de las clases son lógicamente posibles pero empíricamente imposibles. 
(2) utilizando otro equipo diseñado para el mismo fin. En este caso, es posible que el experimento:

(2.1) mantenga constante el diseño experimental del experimento original y el cuerpo teórico presupuesto. Es el caso de la replicación de experimentos; (2.2) varíe en su diseño, en cuyo caso los presupuestos teóricos pueden:

(2.2.1) permanecer constantes. El experimento será del mismo tipo que el original. Podemos denominar a este caso de reproducción T-repetición. (En este caso, el prefijo T indica que lo que permanece constante es el cuerpo teórico presupuesto);

(2.2.2) modificarse. Las teorías presupuestas en el diseño del experimento cambian respecto de las supuestas en el experimento original, con lo que estaríamos frente al caso de la contrastación independiente.

Podemos señalar, en principio, dos sentidos en los que puede hablarse de contrastación independiente. En un primer sentido, se supone que los experimentos funcionan a partir de procesos físicos no relacionados causalmente y presuponen teorías lógicamente independientes. ${ }^{10}$ En un segundo sentido, una versión debilitada de la contrastación independiente exige que la misma dependa de propiedades diferentes del objeto que se investiga. En cualquiera de los casos, esta forma de reproducción, si bien tiene alta incidencia en el momento de validar un resultado experimental, no constituye la repetición de un experimento, sino un nuevo procedimiento para medir una magnitud o parámetro determinados, es decir una reproducción.

Hemos presentado aquí un esbozo de clasificación de los modos de reproducción experimental. Teniendo en cuenta el objetivo primario de este trabajo, no avanzaremos en este tema; sin embargo, cabe señalar que la I-repetición y la replicación son casos especiales de la T-repetición. La T-repetición involucra la variación tanto del instrumento empleado para la medición, como de la sensibilidad del mismo; la I-repetición, por su parte, es un caso en el que la variación del instrumento de medición y de la

10 La posibilidad de contrastación independiente en sentido fuerte, es decir, en el sentido de que las teorías supuestas en el diseño experimental sean lógicamente independientes, supone la evaluación previa de un conjunto de tesis respecto de la naturaleza de las teorías científicas, que no podemos profundizar en este trabajo. Sin embargo, en la mayor parte de los casos, la independencia que puede establecerse es más débil; en general, los diseños experimentales toman como punto de partida propiedades diversas de aquello que se intenta medir, por ejemplo, en la medición de la tasa de neutrinos solares, entendemos como una contrastación independiente del experimento original propuesto por Davis, a las mediciones llevadas a cabo en el Sundbury Neutrino Observatory. El primer experimento tomó en consideración la interacción débil de los neutrinos, mientras que el segundo, consideró el efecto Cerenkov (cf. Franklin, 2004). 
sensibilidad son iguales a cero; mientras que en la replicación hay variación del instrumento de medición - sólo en el sentido en que es otro el instrumento utilizado - pero no de sensibilidad. Baste lo dicho a modo de ilustración de la multiplicidad de los modos de reproducción experimental. ${ }^{\mathbf{1 1}}$ Nos detendremos en lo que sigue en la noción de replicación, buscando precisar más éste concepto.

\section{LA REPLICACIÓN DE EXPERIMENTOS Y EL GONOGIMIENTO TÁGITO}

Según Franklin, dos experimentos son réplicas si se cumple que miden "la misma magnitud física" (1998, p. 155). Por el contrario, nosotros entendemos que la medición de una misma magnitud si bien es una condición necesaria para que dos experimentos se consideren réplicas, de ningún modo es suficiente. Tal como se desprende de la clasificación que presentamos, es una condición necesaria para la repetición en general y, más aun, para la reproducción de todo resultado experimental.

Para el caso específico en el que se intenta reproducir un resultado experimental por medio de la replicación de un experimento, sumamos a la exigencia de medir una misma magnitud, la constancia en el diseño experimental y en el cuerpo teórico presupuesto. Entendemos que los tres requisitos son necesarios para considerar que un experimento es una réplica de otro. Sin embargo, no son suficientes, dado que es posible que ambos experimentos los cumplan y no sean réplicas más que aparentemente. Intentaremos a continuación contextualizar el problema de la replicación de experimentos, mostrando por qué los requisitos anteriores no bastan para definir este concepto y propondremos una condición adicional que (entendemos) permitirá caracterizar de manera suficiente el concepto en cuestión.

El término réplica, si seguimos su uso en las ciencias biomédicas, lleva asociada la connotación de identidad. La replicación es el mecanismo biológico por medio del cual una molécula de DNA se reproduce, dando como producto de dicho proceso otra molécula idéntica a la primera, es decir, idéntica respecto de sus propiedades moleculares pero numéricamente diferente. Entendemos, además, que es este el sentido que se da al término en los trabajos canónicos de diseño experimental. ${ }^{\mathbf{2}}$ Por ello, creemos

11 En trabajos próximos, nuestro objetivo será desarrollar una teoría de la reproducción experimental que avance sobre esta clasificación y que evalúe cada modo de reproducción desde una perspectiva epistemológica.

12 Debemos destacar, sin embargo, que podemos hallar dos formas de replicación en la práctica científica. Una interna, y otra externa. La primera forma de replicación es muy habitual y es parte de un mismo experimento, por ejemplo, cuando se evalúan los efectos de una sustancia en varios roedores, o cuando se estudian ciertas propiedades en un tipo de partícula. En este trabajo nos detenemos en la segunda forma de replicación, que tiene como meta replicar un experimento previo. 
que es esta característica la que debemos privilegiar al momento de definir la noción de replicación para los experimentos en ciencias fácticas, siendo ahora fundamental establecer a qué tipo de identidad estamos aludiendo. Resulta llamativo que los autores que participan en esta discusión asignen significados tan diversos al término réplica. Collins enfatiza alguna forma de identidad entre detectores, al punto de considerar que dos instrumentos son réplicas en el caso de ser isomórficos (cf. Collins, 1992, p. 170-1). Asumimos que, para que la discusión sea fructífera, es preciso un acuerdo respecto del significado de los términos utilizados. Sin embargo, Radder, aun al discutir el argumento del regreso del experimentador, da un sentido completamente diferente al término réplica. Según su propuesta, dos experimentos son réplicas si reproducen un resultado con independencia del conocimiento teórico presupuesto (Radder, 1995, p. 66). Sería, entonces, deseable, tanto filosófica como metodológicamente, establecer un uso canónico de los términos técnicos involucrados en la reproducción experimental.

Si la replicación de un experimento tiene como objetivo la contrastación de un resultado experimental y, por tanto, la importancia de la réplica reside en la posibilidad de contrastar los resultados del instrumento original, aquello que resulta necesario y que, en conjunción con los requisitos antes mencionados de invariancia teórica e invariancia del diseño experimental, caracteriza de manera suficiente a una réplica es el hecho de que ambos instrumentos sean funcionalmente idénticos, ${ }^{\mathbf{1 3}}$ es decir, que, dada una misma señal emitida por la fuente estudiada, ambos instrumentos proporcionen un dato coincidente. Sin embargo resulta de dudosa utilidad práctica la postulación del requisito de identidad funcional ya que, para que el juicio respecto del éxito en la replicación pueda formularse, es preciso proponer un método para evaluar si dos dispositivos son o no funcionalmente idénticos. A continuación propondremos una posible forma de determinar tal identidad.

La calibración, el uso de una señal sustituta con el objetivo de estandarizar un instrumento (cf. Franklin, 1998, p. 237), es un recurso fundamental a la hora de evaluar si un instrumento de medición funciona apropiadamente. Una característica peculiar del proceso de calibración es que la señal, que habitualmente se considera el output de la medición, funciona aquí como el valor conocido que permitirá ajustar el instrumento. Todos los instrumentos utilizados en la práctica científica deben ser pe-

13 Cabe la pregunta respecto de por qué apelamos a la identidad funcional y no a la material, como podría esperarse. Que la identidad material no es suficiente para la replicación puede entenderse a partir de un ejemplo de la química. Los enantiómeros son sustancias materialmente idénticas, sin embargo, al diferir respecto de la disposición espacial de los átomos constituyentes, son imágenes especulares no superponibles. Como consecuencia, la actividad óptica de las sustancias difiere. 
riódicamente calibrados ya que esta operación permite eliminar el error de ajuste, un subtipo de error sistemático. Pero, no es sólo en virtud de evitar el error de ajuste que la calibración es relevante antes de la toma de datos, sino también porque un instrumento de medición es un sistema físico y, como tal, se ve afectado por el desgaste de sus partes, lo que incide en su correcto funcionamiento.

Ahora bien, la calibración supone conocer y poder manipular la fuente que se intenta investigar. Dado que en la investigación de frontera es generalmente imposible satisfacer este requisito, la calibración del instrumento se realiza postulando una similitud entre la fuente a investigar y otra señal ya conocida. En los intentos de detección de ondas de gravedad, algunos de los equipos se calibraron con pulsos de energía acústica (cf. Franklin, 1998, p. 245). Sin embargo, que el detector pueda captar adecuadamente la señal sustituta no nos autoriza a afirmar que detecta adecuadamente la señal desconocida, dado que la proposición, que afirma la similitud entre señales, podría ser falsa. Pero si bien no es posible apelar a esta estrategia como medio para juzgar el buen funcionamiento de un instrumento, la calibración puede resultar un medio útil para determinar si un detector es o no una réplica adecuada del original, permitiendo conjeturar la identidad funcional entre los instrumentos sometidos a comparación. En este caso, la calibración adoptará la forma de una calibración múltiple. Si los detectores son expuestos a un conjunto de inputs y proveen outputs coincidentes para los mismos, podemos inferir de manera inductiva que para aquella señal desconocida se comportarán de igual modo. Asimismo, podremos inferir que la transmisión de conocimiento tácito ha sido exitosa. Ejemplificando lo anterior a partir del caso que hemos estudiado en las páginas precedentes, podemos decir, mutatis mutandis, que el detector utilizado por Weber y aquel utilizado por Garwin podrían considerarse funcionalmente idénticos, si siendo sometidos a una fuente de pulsos acústicos de intensidad conocida, fuesen ambos capaces de registrar el mismo valor para los pulsos y, si al ser excitados por una fuente electrostática común, respondiesen de igual modo. Esto es meramente ilustrativo. No sostenemos que efectivamente las antenas de ambos investigadores sean réplicas, por cuanto difieren en su sensibilidad. Contrariamente a lo que sostiene Franklin (1998), creemos que esos experimentos son T-repeticiones.

El criterio de identidad funcional, si bien estimo que no es inmune a la crítica, provee un recurso útil en el momento de juzgar la transmisión exitosa de conocimiento tácito y, como veremos a continuación, permite ofrecer una respuesta al argumento escéptico de Collins. 


\section{LAS RESPUESTAS AL ARGUMENTO}

El argumento del regreso del experimentador ha sido discutido en extenso tanto por epistemólogos y sociólogos de la ciencia como por científicos. Entre las diversas respuestas, es particularmente interesante la que proporciona Franklin en diversos trabajos (cf. 1998, 1999, 2002). Podríamos pensar que su crítica es básicamente de carácter histórico, ya que se ocupa con detalle del análisis del caso de las ondas de gravedad para mostrar cómo la comunidad científica, al rechazar los resultados de Weber, utilizó criterios racionales. Según su reconstrucción, Weber cometió un conjunto de errores en el transcurso de sus experimentos que disminuyeron la credibilidad de los resultados obtenidos. Por ejemplo, la coincidencia de pulsos entre los dos detectores era consecuencia de una sincronización incorrecta, la correlación sideral propuesta era espuria, la elección de la técnica para el procesamiento de las vibraciones en el instrumento no era la adecuada, el umbral a partir del cual un pulso se consideraba una onda de gravedad variaba en función de los datos obtenidos, los detectores no eran calibrados, el aumento de la sensibilidad del detector no generó una modificación de la proporción entre ruido y señal y, finalmente, no hubo corroboración por parte de otros grupos de investigación autónomos.

Si bien el aporte de Franklin es sumamente valioso, entendemos que resulta conveniente trascender los límites propios de la reconstrucción de casos empíricos. Por ello, en lo que sigue, intentaremos ofrecer una respuesta de carácter filosófico, una respuesta que, por tanto, no se limite a mostrar por qué el ARE no tuvo lugar en el caso de las ondas gravitatorias, sino por qué el ARE no es un problema para la práctica experimental en general.

Tras haber elucidado la noción de replicación en las líneas anteriores, comenzaremos por ofrecer una respuesta al ARE en su versión epistemológica.

Collins nos instaba a aceptar que la replicación constituía el único medio legítimo para la contrastación de un enunciado empírico en la investigación de frontera y, tal como se desprendía de nuestro análisis de ARE 1, esta forma de repetición experimental involucraba conocimiento tácito, lo que volvía imposible conocer si un instrumento era o no una réplica de aquel cuyos resultados se sometían a evaluación. Si aceptamos que el método que hemos propuesto para establecer si dos instrumentos son réplicas, esto es, el método de la calibración múltiple para determinar la identidad funcional, y aceptamos también que dos instrumentos funcionalmente idénticos lo son en virtud de la transferencia exitosa de conocimiento tácito, estaríamos en condiciones de decidir en qué casos un experimento puede considerarse una réplica adecuada y, por consiguiente, que, aun cuando la replicación fuese la única estrategia posible para contrastar un resultado experimental, es factible decidir si el conocimiento 
tácito ha sido transferido, con lo que el regreso al infinito puede evitarse con medios internos a la práctica científica.

Desde luego, esta propuesta no está exenta de inconvenientes. ${ }^{\mathbf{1 4}}$ El criterio de identidad funcional, como posible método de evaluación del éxito en la replicación y de la transferencia de conocimiento tácito, se vale de una inducción que, en muchos casos, puede no ser justificada. ¿Cuál es el número de señales en que deben coincidir los detectores comparados para evitar una inducción apresurada? Es este un problema que debería evaluarse en cada situación experimental, si se desea utilizar la identidad funcional como una estrategia que permita superar los problemas planteados por la primera versión del argumento.

Pero, por otra parte, Collins señaló, como hemos indicado oportunamente, una asimetría entre los recursos confirmatorios y los disconfirmatorios a partir de la cual exigía que, en todo intento de disconfirmar un resultado experimental problemático, se apelase a la replicación. Teniendo en cuenta nuestra caracterización de la noción de replicación y tomando seriamente la exigencia de Collins, llegamos a la paradójica consecuencia de que, si las réplicas son tales, confirmarán inevitablemente los resultados previos. Con ello, asumimos que la replicación de experimentos, lejos de ser como Collins pretendiera, el único medio epistemológicamente aceptable para la reproducción de un resultado experimental, no permite dirimir el estatus de un resultado conflictivo. Franklin y Howson (1984) sostienen, a partir del acercamiento bayesiano al problema, que la replicación de experimentos posee escaso valor confirmatorio cuando es comparada con otros procedimientos para la reproducción de resultados. Coincidimos con ellos agregando que la replicación experimental, por sus características, tampoco permitirá la disconfirmación.

Llegamos entonces a la conclusión de que el problema no reside en las dificultades de la replicación, algo que es consistente con la historia del caso que hemos presentado. Por el contrario, el problema reside en cómo determinar qué propiedades posee una señal que es poco conocida, un aspecto que cobra especial relevancia en la investigación de frontera.

Con lo anterior, pretendemos haber mostrado las tres primeras tesis que nos permitirían eludir el regreso pretendido por Collins:

14. Si bien consideramos que la identidad funcional es una condición necesaria y suficiente para que dos instrumentos sean réplicas, dado que la determinación de la identidad funcional se logra apelando a procedimientos empíricos, la postulación de identidad funcional es, como toda proposición de carácter fáctico, conjetural. No obstante, la calibración múltiple aporta buenas razones para aceptar que dos instrumentos son réplicas. 
(1) la replicación no es el medio exclusivo para la contrastación de un resultado experimental;

(2) La calibración múltiple permite conjeturar la identidad funcional entre instrumentos y, con ello, la transferencia de conocimiento tácito;

(3) En sentido estricto, consideramos que la replicación, tal como ha sido definida, no es un medio epistemológicamente viable para la contrastación en la investigación de frontera.

Creemos, al margen de las razones filosóficas que hemos aportado para realizar tal afirmación, encontrar sustento histórico para nuestra tesis. Difícilmente dos equipos de investigación replican experimentos; por el contrario, la norma en la práctica científica es la variación de los procedimientos experimentales que, en general, tienen como consecuencia un aumento de sensibilidad respecto del experimento original y que, según nuestra clasificación, son casos de T-repeticiones. Un caso que es comparable con el de las ondas gravitatorias tanto por la polémica que generó en la comunidad física como por las dificultades del experimento es el de la posibilidad de inducir reacciones nucleares a bajas temperaturas: la fusión en frío. En este episodio, Martin Fleischmann y Stanley Pons afirmaron haber logrado la fusión nuclear a partir de átomos de deuterio. ${ }^{15} \mathrm{Al}$ tiempo, intentando reproducir los resultados de Fleischmann, Abriola y su equipo construyeron un dispositivo 25 veces más sensible en el que no encontraron evidencia alguna de la emisión de neutrones a 2.5 MeV, valor del resultado informado por Fleischmann y Pons (cf. McKinney, 1998). Encontramos aquí otro ejemplo de un caso conflictivo en el que, lejos de replicar el experimento sometido a evaluación, se busca reproducir el resultado apelando a instrumentos mucho más sofisticados y sensibles.

Hay, sin embargo, casos de experimentos replicados cuyo objetivo no es ni la confirmación ni la disconfirmación de un resultado previo, sino que se proponen como replicaciones de interés histórico por cuanto están destinados a evaluar si un científico realizó o no cierto procedimiento, si un experimento pudo haber dado o no cierto resultado. Como ejemplo, podemos pensar en las replicaciones de los experimentos de Galileo que llevaron a cabo de manera independiente Drake (1973), Naylor (1980) y Hill (1986) o, tomando un artículo reciente sobre el tema, en la replicación del kaliapparat de Justus Von Liebig, instrumento destinado al análisis de sustancias orgánicas (cf. Usselman; Reinhart \& Foulser, 2005).

15 La fusión nuclear es un proceso que tiene como resultado una liberación extraordinaria de energía, y de poder lograrse a bajas temperaturas tendría un impacto económico de extrema importancia. 
En resumen, aun si la replicación fuese el único medio para la contrastación de un resultado controvertido, sería posible transferir el conocimiento tácito; sin embargo, la replicación no es un medio legítimo para tal fin, ergo, la versión ARE 1 no plantea un desafío serio para la racionalidad de la ciencia experimental.

Evaluaremos ahora la versión ontológica del ARE para concluir nuestra discusión. Pero antes, se vuelve necesaria una serie de aclaraciones respecto de las relaciones entre las dos versiones del argumento.

Como mencioné en un apartado anterior, Collins asume que ARE 2 es un caso particular de ARE ı. No obstante, creemos que los argumentos son independientes. Mientras que ARE 1 indica de qué modo reproducir un resultado experimental, ARE 2 indica los problemas que pueden presentarse al intentar establecer el resultado correcto. Quebrar el regreso al infinito propuesto por ARE 1 nos permite mostrar que es posible la reproducción experimental y que la replicación es sólo uno de los tantos modos que la reproducción puede adoptar, pero no nos autoriza a afirmar que dada una reproducción exitosa - aquella en la que los resultados obtenidos coincidan -, estos resultados sean correctos respecto de los fenómenos. La reproducción exitosa es sólo uno de los aspectos en el contexto de la epistemología de la experimentación que nos permiten evaluar la validez de un resultado (cf. Franklin, 1989). Evitar la circularidad propuesta por ARE 2 supondrá, entonces, mostrar que es posible establecer el resultado correcto, a partir de criterios internos, teniendo en cuenta la falibilidad intrínseca del conocimiento científico.

Como anticipamos, la versión ontológica presupone la aceptación de compromisos epistemológicos cuestionables. Al formularla, Collins asume que el único medio para determinar el resultado correcto es la previa determinación del buen funcionamiento de un instrumento de detección y, con ello, presupone la aceptación de una versión extrema de la tesis de la autonomía de la experimentación por cuanto asume la posibilidad de un acceso independiente al mundo físico, un acceso libre de mediación teórica. Sin embargo, es dudoso que un experimento en la investigación de frontera carezca de lineamientos teóricos, por el contrario, los lazos entre teoría y experimentación se estrechan en estos casos y, en general, la coincidencia con las predicciones teóricas es un indicador del buen funcionamiento del instrumento empleado en la medición. Coincidimos con Kuhn en este punto, cuando afirma:

En los casos en que la medición es insegura, una de las pruebas de confiabilidad de los instrumentos existentes y de las técnicas de manipulación ha de consistir, inevitablemente, en su capacidad para dar resultados que concuerden favorablemente con la teoría existente. En algunas partes de la ciencia natural, sólo de esta manera puede juzgarse la adecuación de la técnica experimental (Kuhn, 1977, p. 209). 
La determinación del resultado correcto en casos problemáticos puede establecerse a partir de las teorías mejor confirmadas en la disciplina en cuestión y, entonces, aceptaremos que el instrumento funciona correctamente, si es capaz de proveer resultados que coincidan con las expectativas teóricas. En el caso estudiado, Weber cometió una serie de errores (cf. Franklin, 2002) y, además, obtuvo resultados que requerían, de acuerdo con la teoría general de la relatividad, la producción de efectos observables que no se observaron oportunamente. Como hemos señalado, los resultados de Weber, de ser correctos, debían estar acompañados por fenómenos cosmológicos visibles a ojo desnudo (cf. Levine, 2004).

Sin embargo, convertir lo anterior en una norma universal de aplicación irrestricta traería algunas consecuencias indeseables. Aplicada como imperativo en la actividad científica, el cambio teórico resultaría imposible y, aceptada como regla epistemológica sin excepción, traería como consecuencia la adopción de una posición filosófica que no es capaz de explicar el cambio científico. En consecuencia, la aceptación de una epistemología descriptivamente inadecuada. Por ello, creemos que la apelación al contexto teórico es lícita sólo si las teorías a partir de las cuales determinaremos el resultado correcto han sido anteriormente bien confirmadas y, desde luego, si no son modificadas de manera $a d$ hoc a fin de alcanzar tal coincidencia. En este sentido, creemos justo defender que la concordancia de los resultados de Weber con las predicciones de la teoría de la relatividad general, que cumple notablemente con la imposición anterior (cf. Will, 1993, 2006), podría tomarse como un indicador del resultado a esperar y, con ello, del buen funcionamiento del instrumento de detección. En casos menos claros, entendemos que no resulta pertinente tomar la coincidencia entre una predicción teórica y el resultado obtenido como criterio para establecer la idoneidad del instrumento.

Pero aun en aquellos casos en los que la teoría no cumple con el requisito antes impuesto, es posible, con el fin de determinar cuál sea el resultado correcto, apelar a vías alternativas más seguras, sea a mediciones indirectas o bien a la coincidencia con los resultados de experimentos independientes. En el caso que presentamos, una medición indirecta, que podría favorecer la hipótesis de la existencia de ondas gravitatorias, es la medición de la frecuencia orbital de los púlsares binarios (cf. Will, 1993), mientras que el ejemplo representativo para la contrastación independiente de los resultados de Weber podríamos ejemplificarlo con la medición de la radiación gravitatoria que utilizó a la Tierra como detector (cf. Wiggins \& Press, 1969). Sin duda alguna, determinar el resultado correcto, a partir de la coincidencia entre predicciones teóricas y/o a partir de la coincidencia de los resultados provistos por experimentos independientes, es una apelación solapada al argumento del no-milagro propuesto por Putnam en su época realista (cf. Putnam, 1971; Salmon, 1984) y, como tal, estará sujeto a las 
críticas que recibiera el mismo. Se trata, sin duda, de un argumento no concluyente. Creemos, sin embargo, que pretender conclusiones apodícticas respecto de problemáticas empíricas es reclamar un grado de certeza a todas luces inaccesible.

Para concluir, respecto de la cuarta tesis que presentamos como respuesta posible al ARE, defendemos que el resultado correcto puede establecerse con criterios independientes al funcionamiento del instrumento y, por ello, creemos haber mostrado que la versión ARE 2 no representa un problema insoluble para la racionalidad de la práctica científica.

\section{Conclusiones}

Podemos resumir nuestras conclusiones de la siguiente manera:

(1) Una réplica es tal que al ser comparada con el instrumento replicado se cumple que: ambos instrumentos miden la misma magnitud física, ambos suponen para su funcionamiento el mismo cuerpo de conocimiento teórico, el diseño experimental permanece inalterado y, finalmente, los instrumentos comparados son funcionalmente idénticos.

(2) La identidad funcional entre detectores puede inferirse a partir del método de calibración múltiple.

(3) La identidad funcional es un indicador de la transferencia exitosa de conocimiento tácito.

(4) Por sus características, experimento replicador y experimento replicado no pueden más que ofrecer valores coincidentes de las magnitudes medidas, con lo que la replicación no resulta - a diferencia de lo sostenido por Collins - un medio epistemológicamente adecuado para dirimir el status de un resultado experimental conflictivo.

Mostramos así que la versión epistemológica del ARE descansa en presupuestos falsos y, con ello, no plantea un desafío serio a la racionalidad en la actividad experimental. Continuamos reflexionando respecto del alcance de la versión ontológica del argumento, ARE 2, considerando relevante la pregunta acerca de los medios que nos permitirían juzgar cuál es el resultado correcto de un experimento y proseguimos nuestra investigación indicando que:

(5) ARE 2 supone la adhesión a una tesis extrema de la autonomía de la experimentación. 
(6) La autonomía de la experimentación, así entendida, supone que el experimento no se sustenta en presupuestos teóricos que permitan establecer $a$ priori qué clase de resultado es esperado.

(7) Las teorías científicas, que han sido objeto de contrastaciones previas, que han realizado predicciones novedosas posteriormente corroboradas y son consideradas por la comunidad científica como conocimiento fuera de toda duda razonable en un momento determinado, permiten evaluar la pertinencia de un resultado experimental, indicando cuál sería el resultado correcto en caso de que la teoría fuese verdadera.

(8) Si la teoría o conjunto de teorías de la cual la predicción del resultado se infiere no cumple con los requisitos anteriores, las mediciones indirectas y la contrastación independiente son vías a las que puede apelarse con el fin de determinar si el resultado provisto por un experimento es o no correcto y, con ello, si el instrumento que lo produjo funciona adecuadamente.

(9) La coincidencia entre resultados de diversas mediciones es un argumento abductivo que proporciona razones para juzgar cuál es el resultado correcto.

Ahora bien, en tanto una teoría científica, aun siendo considerada conocimiento aceptado en un momento dado, es siempre susceptible de una refutación posterior y, dado que la coincidencia entre múltiples mediciones de una misma magnitud por medios alternativos es una forma del argumento del no-milagro y, con ello, un tipo de razonamiento abductivo, es decir, deductivamente inválido, no nos es lícito considerar a los productos de la experimentación como conocimiento infalible. Pero ello, sin embargo, tampoco nos autoriza a afirmar, como pretende Collins, que los fundamentos del conocimiento empírico sean meramente el producto de la persuasión o la negociación. Simplemente debemos asumir que el conocimiento experimental posee el mismo carácter hipotético y, por tanto, provisorio que hemos atribuido a toda teoría empírica. Si aceptamos lo anterior, estaremos reduciendo la pretensión de objetividad extrema que condena al escepticismo y al relativismo a quienes tan altas exigencias reclaman al conocimiento científico.

En este trabajo, hemos intentado dar respuesta al desafío escéptico relativista de Collins, y defender que la práctica experimental, aun cuando no está en condiciones de procurar certezas, produce conocimiento confiable, si bien revisable. Pero además, creemos haber puesto de manifiesto la necesidad de procurar una mejor comprensión del proceso de producción del conocimiento experimental. Esta discusión, entonces, debe concebirse como el primer paso para el desarrollo de un programa de investigación en el contexto de la filosofía de la ciencia, un programa que, además de los temas tratados en este trabajo, permita la elaboración de una teoría de la individuación de 
experimentos que precise las variadas funciones que pueden cumplir en la práctica científica que permita establecer, de manera precisa, los vínculos entre experimentos y teorías presupuestas y que, finalmente, investigue el valor epistemológico de cada forma de reproducción experimental.

Agradecimientos. Este trabajo es resultado de mis investigaciones en calidad de becaria doctoral de Conicet (Consejo Nacional de Investigaciones Científico y Técnicas). Una versión previa del artículo fue distinguida con el "Premio Rabossi" (2008) de Sadaf (Sociedad Argentina de Análisis Filosófico). Quiero agradecer especialmente a Alejandro Cassini y a José Díez por sus valiosas observaciones y sugerencias.

\title{
Romina Zuppone
}

Becaria doctoral de la Universidad de Buenos Aires, Consejo Nacional de Investigaciones Científicas y Técnicas, Argentina. romina@filo.uba.ar

\begin{abstract}
The aim of this article is to analyze and criticize the argument of the experimenter's regress proposed by Harry Collins in 1985 . To this end, we begin by discussing the experiment that aimed to detect gravity waves performed by Joseph Weber during the 1970s. We continue with analysis and discussion of both the epistemological and ontological versions of Collin's argument. Then, finally, after giving an outline of a theory of experimental reproduction and an explication of the concept of replication, we propose two ways of avoiding the experimenter's regress.
\end{abstract}

KEYWORDS • Experimenter's regress. Experiment. Experimental reproduction. Replication.

Collins. Weber.

\section{REFERENGIAS BIBLIOGRÁFIGAS}

Ackermann, R. Data, instruments, and theory: a dialectical approach to understanding science. Princeton: Princeton University Press, 1985.

Barnes, J. The toils of skepticism. Cambridge: Cambridge University Press, 1990.

Buchtwald, J. (Ed.). Scientific practice: theories and stories of doing physics. Chicago: University of Chicago Press, 1995 .

CARTwRight, N. Replicability, reproducibility and robustness: comments on Harry Collins. History of Political Economy, 21, p. 143-55, 1991.

Collins, H. The seven sexes: a study in the sociology of a phenomenon, or the replication of experiments in physics. Sociology, 9, p. 205-24, 1975.

. Son of the seven sexes: the social destruction of a physical phenomenon. Social Studies of Science, 11 , p. 33-62, 1981 . 
Collins, H. Changing order: replication and induction in scientific practice. Chicago: University of Chicago Press, 1985 .

. The meaning of replication and the science of economics. History of Political Economy, 21, p. 12342, 1991.

. Changing order: replication and induction in scientific practice. 2. ed. Chicago: University of Chicago Press, 1992.

. A strong confirmation of the experimenters' regress. Studies in History and Philosophy of Modern Physics, 25, 3, p. 493-503, 1994 .

. Tacit knowledge, trust and the Q of sapphire. Social Studies of Science, 31, p. 71-85, 2001.

. The experimenters' regress as philosophical sociology. Studies in History and Philosophy of Science, 33 , p. $15^{3-60,2002 .}$

. Gravity's shadow: the search for gravitational waves. Chicago/London: University of Chicago Press, 2004 .

Culp, S. Objectivity in experimental enquiry: breaking data-techniques circles. Philosophy of Science, 62 , p. $430-50,1995$.

Davies, P. En busca de las ondas de gravitación. Barcelona: Salvat, 1987 [1980].

Drake, S. Galileo's exprimental confirmation of horizontal inertia: unpublished manuscripts. Isis, 64, p. 291-3०5, 1973.

Franklin, A. The epistemology of experiment. In: Gooding, D.; Pinch, T. \& Schaffer, S. (Ed.). The uses of experiment: studies in the natural sciences. Cambridge: Cambridge University Press, 1989. p. 437-60. . Avoiding the experimenters' regress. In: Koertge, N. (Ed.). A house built on sand: exposing postmodernist myths about science. Oxford: Oxford University Press, 1998. p. 151-65.

. Can that be right? Essays on experiment, evidence, and science. Dordrecht/Boston/London: Kluwer Academic Publishers, 1999.

. Selectivity and discord: two problems of experiment. Pittsburgh: University of Pittsburgh Press, 2002. Are there really neutrinos? An evidential history. Cambridge: WestView Press, 2004.

Frankinn, A. \& Howson, C. Why do scientists prefer to vary their experiments? Studies in History and Philosophy of Science, 6, p. 14,1-84, 1984.

Garwin, R. \& Levine, J. New negative results for gravitational wave detection, and comparison with reported detection. Physical Review Letters, 33, 13, p. 794- 7 , 1974 .

Godin, B. \& Gingras, Y. The experimenters'regress: from skepticism to argumentation. Studies in History and Philosophy of Science, 33, p. 133-4,8, 2002.

Gooding, D.; Pinch, T. \& Schaffer, S. (Ed.). The uses of experiment: studies in the natural sciences. Cambridge: Cambridge University Press, 1989.

Hacking, I. Representing and intervening. Cambridge: Cambridge University Press, 1996 [1983].

. The self-vindication of laboratory sciences. In: Pickering, A. (Ed.). Science as practice and culture. Chicago/London: University of Chicago Press, 1992. p. 29-64.

HiLl, D. Dissecting trayectories: Galileo's early experiments on projectiles motion and the law of fall. Isis, 79, p. 646-68, 1986.

Hones, M. Reproducibility as a methodological imperative in experimental research. PSA, 1, p. $5^{8} 5^{-} 99$, 1990.

Koertge, N. (Ed.). A house built on sand: exposing postmodernist myths about science. Oxford: Oxford University Press, 1998.

Knorr-Cetina, K. Relativism - what now. Social Studies of Science, 12, p. 133-6, 1982.

Kunn, T. S. The essential tension. Selected studies in scientific tradition and change. Chicago: University of Chicago Press, 1977 . 
EL ARGUMENTO DEL REGRESO DEL EXPERIMENTADOR Y LA REPLICACIÓN DE EXPERIMENTOS

Latour, B. \& Woolgar, S. Laboratory life: the social construction of scientific facts. London/Beverly Hills: Sage, 1979 .

Laudan, L. A note on Collins blend of relativism and empirism. Social Studies of Science, 12, p. 131-2, 1982 .

Levine, J. Early gravity-wave detection experiments, 1960-1975. Physics in Perspective, 6, p. 42-75, 2004. McKinney, W. When experiments fail: is "cold fusion" science as normal? In: Koertge, N. (Ed.). Ahouse built on sand: exposing postmodernist myths about science. Oxford: Oxford University Press, 1998. p. $133-50$.

Montaigne, M. Essais. Paris: Charpertier, 1862.t. 2.

NAYLOR, R. Galileo's theory of projectile motion. Isis, 71, p. 550-70, 1980.

Pickering, A. (Ed.). Science as practice and culture. Chicago/London: University of Chicago Press, 1992.

Polanyi, M. The tacit dimension. New York: Anchor, 1967.

Popper, K. The logic of scientific discovery. London/New York: Routledge, 1959.

Putnam, H. Philosophy of logic. New York: Harper and Row, 1971.

RAdDER, H. Experimental reproducibility and the experimenters' regress. PSA, 1, p. 63-73, 1992.

Experimenting in the natural sciences: a philosophical approach. In: Buchtwald, J. (Ed.). Scientific practice: theories and stories of doing physics. Chicago: University of Chicago Press, 1995. p. 56-86.

(Ed.). The philosophy of scientific experimentation. Pittsburg: University of Pittsburgh Press, 2003.

Salmon, W. Scientific explanation and the causal structure of the world. Princeton: Princeton University Press, 1984 .

Usselman, M.; Reinhart, C. \& Foulser, K. Restating Leibig: a study in the replication of experiments. Annals of Science, 62, p. 1-55, 2005.

WeBER, J. Detection and generation of gravitational waves. Physical Review, 117, 1, p. 306-13, 1960.

. Gravitational radiation. Physical Review Letters, 18, 13, p. 498-501, 1967.

. Gravitational wave detector events. Physical Review Letters, 20, 23, p. 13o7-8, $1968 \mathrm{a}$.

. Gravitational radiation from the pulsars. Physical Review Letters, 21, 6, p. 395-6, 1968b.

. Evidence for discovery of gravitational radiation. Physical Review Letters, 22, 24, p. 1320-4, 1969.

. Anisotropy and polarization in the gravitational-radiation experiments. Physical Review Letters, 25,

3, p. 180-4, 1970 .

_. Computer analyses of gravitational radiation detector coincidences. Nature, 24, O, p. 28-3o, 1972.

Wiggins, R. \& Press, F. Search for seismic signals at pulsar frecuencies. Journal of Geophysical Research, 74, p. 22, 1969.

WiLL, C. Was Einstein right? Putting general relativity into test. Oxford: Oxford University Press, 1993.

. The confrontation between general relativity and experiment. Living Rev. Relativity, 9, 2006. Accesible en $<3$. URL (http://www.livingreviews.org/lrr-2006-3)>. Acceso en: 20 oct. 2009. 